\title{
Lymphocyte Antigen 75 Polymorphisms Are Associated with Disease Susceptibility and Phenotype in Japanese Patients with Inflammatory Bowel Disease
}

\author{
Atsuhiro Hirayama, ${ }^{1}$ Satoru Joshita, ${ }^{1}$ Kei Kitahara, ${ }^{1}$ Kenji Mukawa, ${ }^{2}$ Tomoaki Suga, \\ Takeji Umemura, ${ }^{1}$ Eiji Tanaka, ${ }^{1}$ and Masao Ota $^{3}$ \\ ${ }^{1}$ Department of Medicine, Division of Gastroenterology and Hepatology, Shinshu University School of Medicine, Matsumoto, Japan \\ ${ }^{2}$ Department of Gastroenterology, Japanese Red Cross Society Suwa Hospital, Suwa, Japan \\ ${ }^{3}$ Department of Legal Medicine, Shinshu University School of Medicine, Matsumoto, Japan
}

Correspondence should be addressed to Satoru Joshita; joshita@shinshu-u.ac.jp

Received 2 September 2016; Accepted 20 October 2016

Academic Editor: Silvia Angeletti

Copyright (C) 2016 Atsuhiro Hirayama et al. This is an open access article distributed under the Creative Commons Attribution License, which permits unrestricted use, distribution, and reproduction in any medium, provided the original work is properly cited.

\begin{abstract}
Recent genome-wide association studies have rapidly improved our understanding of the molecular pathways leading to inflammatory bowel disease (IBD), which includes Crohn's disease (CD) and ulcerative colitis (UC). Although several reports have demonstrated that gene single nucleotide polymorphisms (SNPs) are associated with susceptibility to IBD, its precise genetic factors have not been fully clarified. Here, we performed an association analysis between lymphocyte antigen 75 (LY75) genetic variations and IBD susceptibility or phenotype. SNPs were genotyped in 51 CD patients, 94 UC patients, and 269 healthy controls of Japanese ethnicity. We detected a significant relationship with CD susceptibility for the rs16822581 LY75 SNP $(P=0.045)$. One haplotype (GT, $P=0.042$ ) was also associated with CD susceptibility, while another carrying the opposite SNP (CA) was linked to an absence of surgical history for CD. Our findings confirm that LY75 is involved in CD susceptibility and may play a role in disease activity in the Japanese population.
\end{abstract}

\section{Introduction}

Inflammatory bowel disease (IBD), which includes Crohn's disease (CD) and ulcerative colitis (UC), is a chronic destructive digestive disorder leading to tissue damage, loss of function, disability, and systemic inflammation [1]. CD is characterized by inflammation appearing in any region of the gastrointestinal tract and on the entire wall of the bowel, while UC is restricted to the mucosa of the colon. The number of patients with IBD has been increasing in both developed and developing countries to create clinical and economic problems. It is currently estimated that 1.4 million, 2.2 million, and 0.2 million individuals suffer from IBD in the United States, Europe, and Japan, respectively [2-4]. The precise etiology of this disease group remains poorly understood, although the loss of barrier function in the gut leading to an inappropriate inflammatory response to intestinal microbes [5] and reactivity of infiltrating T cells [6] in genetically predisposed individuals has provided insights into the pathogenesis of IBD [7].

Genome-wide association studies (GWAS) on European populations have uncovered several susceptibility genes to IBD [8] while meta-analyses have identified 71 CD susceptibility loci [9] and 47 UC susceptibility loci [10]. Genes implicated in the type 17 helper T-cell- (Th17-) interleukin23 (IL-23) (Th17-IL23) pathway have been linked to both diseases and the etiology of IBD. Moreover, a meta-analysis of GWAS that analyzed more than 38,000 IBD cases identified an additional 163 susceptibility loci for IBD among European populations [11]. In the Japanese, several susceptibility loci for CD $[12,13]$ and UC [14] were discovered outside of the major histocompatibility complex (MHC) region by GWAS [15]. Based on these reports, multiple, but possibly similar, genes, including those for nucleotide oligomerization domain 
TABLE 1: Demographic and clinical data of CD, UC, and healthy subjects.

\begin{tabular}{lccc}
\hline Characteristic & $\begin{array}{c}\text { CD } \\
(n=51)\end{array}$ & $\begin{array}{c}\text { UC } \\
(n=96)\end{array}$ & $\begin{array}{c}\text { Controls } \\
(n=269)\end{array}$ \\
\hline Age, years & $44(36-52)$ & $49(39-63)$ & $40(34-52)$ \\
Female/male & $15 / 36$ & $43 / 51$ & $205 / 64$ \\
Disease phenotype & & & \\
Location ${ }^{*}$ & $13 / 18 / 17 / 3$ & $44 / 27 / 13 / 10$ & \\
Past surgical history & $20(39)$ & & \\
Presence of anal lesion & $22(43)$ & & \\
\hline
\end{tabular}

Data are expressed as number (\%) except for age, expressed as median (firstthird quartile).

* Location was defined as ileal, colonic, ileocolonic, and not determined in $\mathrm{CD}$ and as proctitis, left-sided colitis pancolitis, and not determined in UC. $\mathrm{CD}$, Crohn's disease; UC, ulcerative colitis.

2 [16] and the Th17-IL23 pathway, have been implicated in CD and UC onset despite ethnicity differences $[17,18]$. Very recently, 38 genetic loci were associated with IBD via a trans-ancestry association study using genome-wide or Immunochip genotype data from an extended cohort of 86,640 European individuals and Immunochip data of 9,846 individuals sampled from ethnicities of East Asian, Indian, or Iranian descent [19], whereby one locus located in the vicinity of lymphocyte antigen 75 (LY75) was reported to be associated with IBD susceptibility [19].

LY75 encodes the endocytic receptor DEC-205, which is a member of the macrophage mannose receptor family of C-type lectins expressed at high levels by $\mathrm{CD}^{+}$dendritic cells (DCs) and thymic epithelial cells $[20,21] . \mathrm{CD}^{+}$DCs expressing DEC-205 play a role in antigen processing and presentation in the context of both MHC class I and MHC class II molecules [20] and generate Thl cell-mediated immune responses in an IL-12-independent, CD70-dependent mechanism $[22,23]$. Therefore, the DEC-205 receptor is suspected to have an important role in T-cell function and homeostasis $[24,25]$.

As associations between LY75 single nucleotide polymorphisms (SNPs) and susceptibility or phenotype have not been investigated in Japanese patients with IBD, this study examined such relationships in Japan.

\section{Patients and Methods}

2.1. Research Ethics Considerations. This study was conducted in accordance with the principles of the 1975 Declaration of Helsinki and approved by the ethics committees of both participating institutions (Shinshu University School of Medicine, Matsumoto, Japan: number 457, and Japanese Red Cross Society Suwa Hospital, Suwa, Japan: number 26-9). Informed written consent was obtained from all participants.

2.2. Subjects. We analyzed a total of 414 subjects (51 CD patients, 94 UC patients, and 269 healthy controls) recruited from Shinshu University Hospital in Matsumoto, Japan, and the Japanese Red Cross Society Suwa Hospital in Suwa, Japan. Subject information is summarized in Table 1 . The participants had no direct relatives of non-Japanese ethnicity, and thus our cohort's racial background was considered to be uniformly Japanese. Control subjects were volunteers from hospital staff who had indicated the absence of any major illnesses and no direct familial relations in a standard questionnaire.

The diagnosis of CD or UC was confirmed by a combination of endoscopic, histopathological, radiological, and biochemical investigations according to existing guidelines $[26,27]$.

We also subdivided the IBD patients into disease parameters based on the phenotypes of disease location (ileal, colonic, ileocolonic, and not determined), history of intestinal resection, and presence of perianal disease for CD and disease location (proctitis, left-sided colitis, pancolitis, and not determined) for UC (Table 1). Regarding extra-intestinal manifestations, only one patient with CD was complicated with polyarthritis and uveitis, while one patient with UC was complicated with polyarthritis.

2.3. SNP Genotyping. Genomic DNA from patients was isolated by phenolic extraction of sodium dodecyl sulfatelyzed and proteinase K-treated cells as described previously and adjusted to a concentration of $10-15 \mathrm{ng} / \mu \mathrm{L}$ [28].

Five SNPs on the LY75 gene (rs1365798, rs13307, rs11690117, rs16822581, and rs1511224) having minor allele frequencies of $>5 \%$ were selected from HapMap Japanese data (https://www.ncbi.nlm.nih.gov/variation/tools/1000genomes/). All SNP genotyping was performed with a TaqMan $5^{\prime}$ exonuclease assay using primers supplied by Applied Biosystems (Foster City, CA, USA). The probe's fluorescence signals were detected with a StepOne Plus Real-Time PCR System (Applied Biosystems) according to the manufacturer's instructions.

2.4. Statistical Analysis. The $\mathrm{R}$ software Haploview version 4.2 [29] was used to evaluate the haplotype structure of the 5 SNPs on LY75. Pairwise linkage disequilibrium (LD) patterns and haplotype frequency analysis for all SNPs in patients and controls were analyzed by the block definition established by Gabriel et al. [30]. We assessed the significance of allele distribution between patients and controls using the $\chi^{2}$ test by means of $2 \times 2$ comparisons $(\mathrm{df}=1)$. A $P$ value of less than 0.05 was considered to be statistically significant. We adjusted $P$ values using Bonferroni's correction $\left(P_{c}\right)$ by multiplication of each locus by 5 . Association strength was estimated by calculating the odds ratio (OR) and 95\% confidence interval (CI). Statistical analysis of findings was performed using IBM SPSS Statistics version 21.0 (IBM, New York, NY, USA) and StatFlex version 6.0 (Artech Co., Ltd. Osaka, Japan) software.

\section{Results}

3.1. LY75 Genotype Analysis of Patients with IBD and Controls. Five SNPs in the LY75 gene (rs1511224, rs16822581, rs11690117, rs13307, and rs1365798) were genotyped in all IBD patients and controls. The genotype frequencies of the tested SNPs were in Hardy-Weinberg equilibrium in all subject groups (Table 2). No statistical differences were found in 
TABLE 2: Allele frequencies of SNPs in the LY75 gene in CD, UC, and healthy subjects.

\begin{tabular}{|c|c|c|c|c|c|c|c|c|c|c|}
\hline \multirow{2}{*}{$\begin{array}{l}\text { SNP } \\
\text { number }\end{array}$} & \multirow{2}{*}{ dbSNP } & \multirow{2}{*}{$\begin{array}{c}\text { Alleles } \\
(1>2)\end{array}$} & \multirow{2}{*}{$\begin{array}{l}\text { Position } \\
\text { (bp) }\end{array}$} & \multirow{2}{*}{$\begin{array}{c}\text { Gene } \\
\text { location }\end{array}$} & \multicolumn{2}{|c|}{$\mathrm{CD}(n=51)$} & \multicolumn{2}{|c|}{$\mathrm{UC}(n=94)$} & \multicolumn{2}{|c|}{ Controls $(n=269)$} \\
\hline & & & & & MAF (\%) & HWE $P$ value & MAF (\%) & HWE $P$ value & MAF (\%) & HWE $P$ value \\
\hline 1 & rs1511224 & $\mathrm{G}>\mathrm{A}$ & 159894350 & Intron & 0.402 & 0.163 & 0.489 & 0.645 & 0.480 & 0.762 \\
\hline 2 & rs16822581 & $\mathrm{C}>\mathrm{T}$ & 159882166 & Exon & 0.431 & 0.221 & 0.335 & 0.619 & 0.329 & 0.893 \\
\hline 3 & rs11690117 & $\mathrm{C}>\mathrm{A}$ & 159841125 & Intron & 0.431 & 0.072 & 0.473 & 0.518 & 0.441 & 0.397 \\
\hline 4 & rs13307 & $\mathrm{C}>\mathrm{T}$ & 159803485 & Intron & 0.402 & 1.000 & 0.346 & 1.000 & 0.357 & 0.922 \\
\hline 5 & rs1365798 & $\mathrm{C}>\mathrm{T}$ & 159795853 & $3^{\prime}$ UTR & 0.186 & 0.254 & 0.282 & 1.000 & 0.307 & 0.704 \\
\hline
\end{tabular}

SNPs, single nucleotide polymorphisms; CD, Crohn's disease; UC, ulcerative colitis; MAF, minor allele frequency; HWE, Hardy-Weinberg equilibrium.

TABLE 3: Allele frequencies of 5 SNPs in CD, UC, and healthy controls.

\begin{tabular}{|c|c|c|c|c|c|c|c|c|c|c|c|c|}
\hline $\begin{array}{l}\text { SNP } \\
\text { number }\end{array}$ & Allele & $\begin{array}{c}\text { CD } \\
(n=51)\end{array}$ & $P$ & $P_{c}$ & OR & $95 \%$ CI & $\begin{array}{c}\mathrm{UC} \\
(n=94)\end{array}$ & $P$ & $P_{c}$ & OR & $95 \%$ CI & $\begin{array}{l}\text { Controls } \\
(n=269) \\
\end{array}$ \\
\hline \multirow{2}{*}{1} & G & 0.60 & \multirow{2}{*}{0.150} & \multirow{2}{*}{0.750} & \multirow{2}{*}{1.37} & \multirow{2}{*}{$0.89-2.11$} & 0.51 & \multirow{2}{*}{0.817} & \multirow{2}{*}{1.000} & \multirow{2}{*}{0.96} & \multirow{2}{*}{$0.69-1.34$} & 0.52 \\
\hline & A & 0.40 & & & & & 0.49 & & & & & 0.48 \\
\hline \multirow{2}{*}{2} & $\mathrm{C}$ & 0.57 & \multirow{2}{*}{0.046} & \multirow{2}{*}{0.230} & \multirow{2}{*}{1.55} & \multirow{2}{*}{$1.01-2.38$} & 0.67 & \multirow{2}{*}{0.878} & \multirow{2}{*}{1.000} & \multirow{2}{*}{1.03} & \multirow{2}{*}{$0.72-1.46$} & 0.67 \\
\hline & $\mathrm{T}$ & 0.43 & & & & & 0.34 & & & & & 0.33 \\
\hline \multirow{2}{*}{3} & $\mathrm{C}$ & 0.57 & \multirow{2}{*}{0.865} & \multirow{2}{*}{1.000} & \multirow{2}{*}{1.04} & \multirow{2}{*}{$0.68-1.59$} & 0.53 & \multirow{2}{*}{0.435} & \multirow{2}{*}{1.000} & \multirow{2}{*}{0.88} & \multirow{2}{*}{$0.63-1.22$} & 0.56 \\
\hline & $\mathrm{A}$ & 0.43 & & & & & 0.47 & & & & & 0.44 \\
\hline \multirow{2}{*}{4} & $\mathrm{C}$ & 0.60 & \multirow{2}{*}{0.386} & \multirow{2}{*}{1.000} & \multirow{2}{*}{1.21} & \multirow{2}{*}{$0.79-1.87$} & 0.65 & \multirow{2}{*}{0.784} & \multirow{2}{*}{1.000} & \multirow{2}{*}{0.95} & \multirow{2}{*}{$0.67-1.35$} & 0.64 \\
\hline & $\mathrm{T}$ & 0.40 & & & & & 0.35 & & & & & 0.36 \\
\hline \multirow{2}{*}{5} & $\mathrm{C}$ & 0.81 & 0.014 & 0.070 & 052 & $030-0.88$ & 0.72 & 0.524 & 1000 & 089 & $062-128$ & 0.69 \\
\hline & $\mathrm{T}$ & 0.19 & & & & & 0.28 & & & & & 0.31 \\
\hline
\end{tabular}

SNPs, single nucleotide polymorphisms; CD, Crohn's disease; UC, ulcerative colitis; $P_{c}$, corrected $P$ value; OR, odds ratio; $95 \%$ CI, $95 \%$ confidence interval.

allele frequencies between patients with $\mathrm{CD}$ or UC and healthy controls (Table 3). However, the genotype frequency of rs16822581 in a recessive model exhibited a statistical difference between $\mathrm{CD}$ patients and controls after adjustment by Bonferroni's correction $(P=0.045)$ (Table 4$)$. No such differences were detected for UC patients and controls (Table 4).

3.2. LY75 Haplotype Analysis between Patients with IBD and Controls. To estimate haplotype frequency and analyze the associations between patients with IBD and healthy controls, tag SNPs were selected using the Tagger algorithm of the Haploview program. Two tag SNPs (rs1511224 and rs16822581) were found to be in strong LD among patients with CD or UC as well as in healthy controls (Figure 1). The top 3 haplotype frequencies in the blocks are shown in Table 5. Haplotype 2 (GT) was significantly associated with CD susceptibility ( $43 \%$ versus $33 \%$; $P=0.042$, OR $1.560,95 \%$ CI 1.014-2.402) as compared with controls.

3.3. Associations between LY75 SNPs, Haplotypes, and Clinical Features in Patients with $C D$. Among the patients with $C D$, $39 \%$ had received prior bowel resection and $43 \%$ had perianal disease (Table 1). However, the allele frequencies of the 5 LY75 SNPs showed no differences compared with controls for the
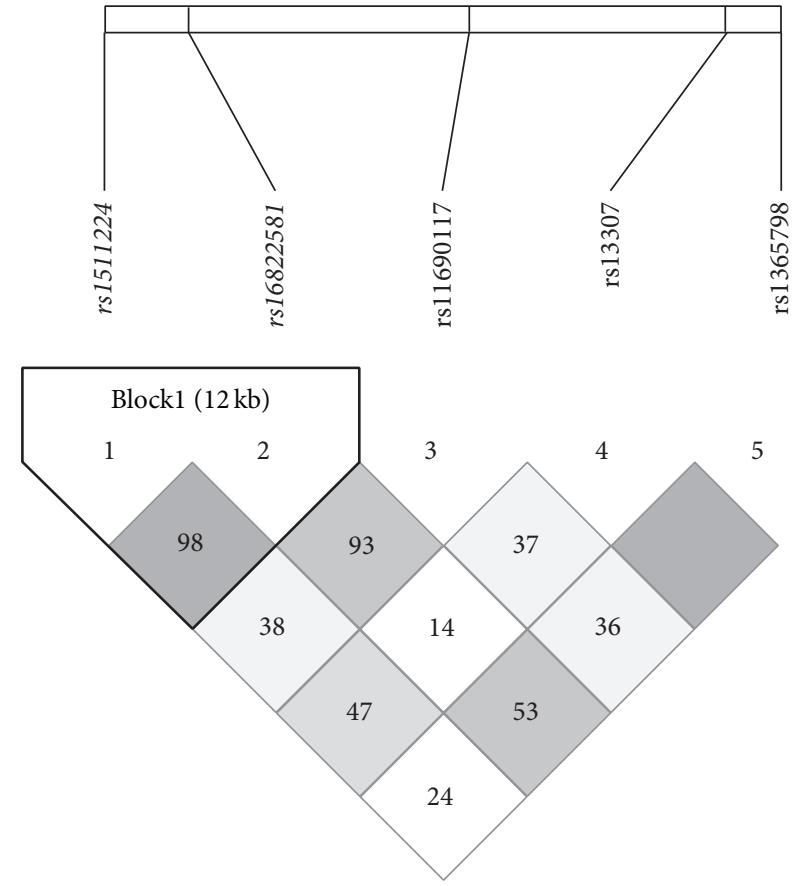

FIGURE 1: Linkage disequilibrium (LD) plot of 5 SNPs in LY75 in 269 healthy subjects. Values of $r 2$ corresponding to each SNP pair are expressed as a percentage and shown within the respective square. 


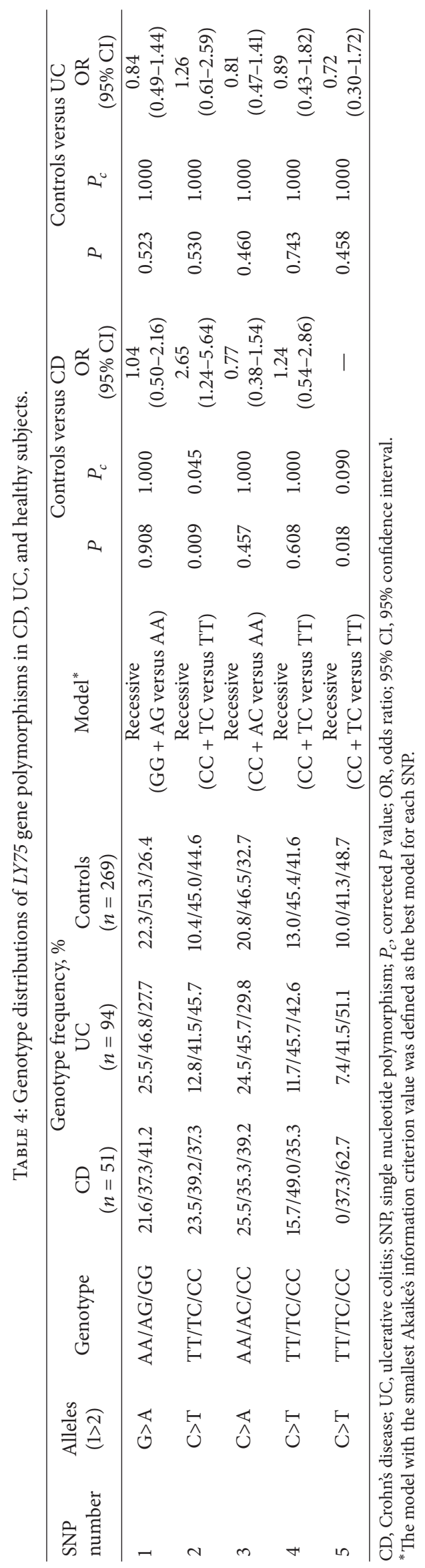


TABLE 5: LY75 haplotypes in CD, UC, and healthy subjects.

\begin{tabular}{|c|c|c|c|c|c|c|c|c|c|c|c|}
\hline \multirow{2}{*}{ Haplotype } & \multicolumn{2}{|c|}{$\begin{array}{c}\text { SNP } \\
\text { number }\end{array}$} & \multicolumn{3}{|c|}{ Frequency } & \multicolumn{3}{|c|}{$\mathrm{CD}$ versus controls } & \multicolumn{3}{|c|}{ UC versus controls } \\
\hline & 1 & 2 & $\begin{array}{c}\mathrm{CD} \\
(n=51)\end{array}$ & $\begin{array}{c}\mathrm{UC} \\
(n=94)\end{array}$ & $\begin{array}{l}\text { Controls } \\
(n=269)\end{array}$ & $P$ & OR & $95 \%$ CI & $P$ & OR & $95 \%$ CI \\
\hline 1 & A & $\mathrm{C}$ & 0.40 & 0.48 & 0.48 & 0.160 & 0.74 & $0.48-1.13$ & 0.881 & 1.03 & $0.74-1.43$ \\
\hline 2 & G & $\mathrm{T}$ & 0.43 & 0.33 & 0.33 & 0.042 & 1.56 & $1.01-2.40$ & 0.947 & 1.01 & $0.71-1.44$ \\
\hline 3 & G & $\mathrm{C}$ & 0.17 & 0.18 & 0.19 & 0.529 & 0.84 & $0.48-1.47$ & 0.708 & 0.92 & $0.60-1.41$ \\
\hline
\end{tabular}

CD, Crohn's disease; UC, ulcerative colitis; SNP, single nucleotide polymorphism; OR, odds ratio; $95 \%$ CI, $95 \%$ confidence interval; $P$ values were calculated by the $\chi^{2}$ test by means of $2 \times 2$ comparisons $(\mathrm{df}=1)$.

TABLE 6: Allele frequencies of LY75 SNPs and phenotypes in patients with CD.

\begin{tabular}{|c|c|c|c|c|c|c|c|c|c|c|c|c|c|}
\hline \multirow[b]{2}{*}{$\begin{array}{l}\text { SNP } \\
\text { number }\end{array}$} & \multirow[b]{2}{*}{ Allele } & \multicolumn{4}{|c|}{ Past surgical history } & \multicolumn{4}{|c|}{ Small intestine lesion } & \multicolumn{4}{|c|}{ Presence of perianal lesion } \\
\hline & & $\begin{array}{c}+ \\
(n=20)\end{array}$ & $\begin{array}{c}- \\
(n=31)\end{array}$ & $P$ & $P_{c}$ & $\begin{array}{c}+ \\
(n=31)\end{array}$ & $\begin{array}{c}- \\
(n=17)\end{array}$ & $P$ & $P_{c}$ & $\begin{array}{c}+ \\
(n=22)\end{array}$ & $\begin{array}{c}- \\
(n=28)\end{array}$ & $P$ & $P_{c}$ \\
\hline \multirow{2}{*}{1} & $\mathrm{G}$ & 0.73 & 0.52 & \multirow{2}{*}{0.036} & \multirow{2}{*}{0.179} & 0.60 & 0.56 & \multirow{2}{*}{0.718} & \multirow{2}{*}{1.000} & 0.55 & 0.64 & \multirow{2}{*}{0.324} & \multirow{2}{*}{1.000} \\
\hline & A & 0.28 & 0.48 & & & 0.40 & 0.44 & & & 0.46 & 0.36 & & \\
\hline \multirow{2}{*}{2} & $\mathrm{C}$ & 0.53 & 0.60 & \multirow{2}{*}{0.475} & \multirow{2}{*}{1.000} & 0.55 & 0.56 & \multirow{2}{*}{0.922} & \multirow{2}{*}{1.000} & 0.59 & 0.54 & \multirow{2}{*}{0.581} & \multirow{2}{*}{1.000} \\
\hline & $\mathrm{T}$ & 0.48 & 0.40 & & & 0.45 & 0.44 & & & 0.41 & 0.46 & & \\
\hline \multirow{2}{*}{3} & $\mathrm{C}$ & 0.60 & 0.55 & \multirow{2}{*}{0.607} & \multirow{2}{*}{1.000} & 0.58 & 0.61 & \multirow{2}{*}{0.767} & \multirow{2}{*}{1.000} & 0.55 & 0.59 & \multirow{2}{*}{0.660} & \multirow{2}{*}{1.000} \\
\hline & A & 0.40 & 0.45 & & & 0.42 & 0.39 & & & 0.46 & 0.41 & & \\
\hline \multirow{2}{*}{4} & $\mathrm{C}$ & 0.65 & 0.57 & \multirow{2}{*}{0.390} & \multirow{2}{*}{1.000} & 0.57 & 0.59 & \multirow{2}{*}{0.822} & \multirow{2}{*}{1.000} & 0.64 & 0.55 & \multirow{2}{*}{0.403} & \multirow{2}{*}{1.000} \\
\hline & $\mathrm{T}$ & 0.35 & 0.44 & & & 0.44 & 0.41 & & & 0.36 & 0.45 & & \\
\hline \multirow{2}{*}{5} & $\mathrm{C}$ & 0.80 & 0.82 & \multirow{2}{*}{0.980} & \multirow{2}{*}{1.000} & 0.81 & 0.85 & 0.771 & 1.000 & 0.77 & 0.86 & 07 & 1.000 \\
\hline & $\mathrm{T}$ & 0.20 & 0.18 & & & 0.19 & 0.15 & & & 0.23 & 0.14 & & \\
\hline
\end{tabular}

SNPs, single nucleotide polymorphisms; CD, Crohn's disease; $P_{c}$, corrected $P$ value.

TABLE 7: Comparison of LY75 haplotype frequencies and phenotypes in patients with CD.

\begin{tabular}{|c|c|c|c|c|c|c|c|c|c|c|c|}
\hline \multirow{2}{*}{ Haplotype } & \multicolumn{2}{|c|}{ SNP number } & \multicolumn{3}{|c|}{ Past surgical history } & \multicolumn{3}{|c|}{ Small intestine lesion } & \multicolumn{3}{|c|}{ Presence of perianal lesion } \\
\hline & 1 & 2 & $\begin{array}{c}+ \\
(n=20)\end{array}$ & $\begin{array}{c}- \\
(n=31)\end{array}$ & $P$ & $\begin{array}{c}+ \\
(n=31)\end{array}$ & $\begin{array}{c}- \\
(n=17)\end{array}$ & $P$ & $\begin{array}{c}+ \\
(n=22)\end{array}$ & $\begin{array}{c}- \\
(n=28)\end{array}$ & $P$ \\
\hline 1 & $\mathrm{~A}$ & $\mathrm{C}$ & 0.28 & 0.48 & 0.036 & 0.40 & 0.44 & 0.718 & 0.46 & 0.36 & 0.324 \\
\hline 2 & G & $\mathrm{T}$ & 0.48 & 0.40 & 0.475 & 0.45 & 0.44 & 0.922 & 0.41 & 0.46 & 0.581 \\
\hline 3 & G & $\mathrm{C}$ & 0.25 & 0.11 & 0.070 & 0.15 & 0.12 & 0.706 & 0.14 & 0.18 & 0.568 \\
\hline
\end{tabular}

$\mathrm{CD}$, Crohn's disease; SNP, single nucleotide polymorphism.

presence or absence of surgical history, small intestine lesions, or perianal disease (Table 6).

Interestingly, haplotype 1 (AC) was significantly negatively correlated with a history of bowel resection in patients with CD (28\% versus $48 \% ; P=0.036)$ (Table 7$)$. No other associations were found for the presence or absence of small intestinal lesions or perianal disease.

\section{Discussion}

The present study investigated the associations between LY75 SNPs and IBD susceptibility or phenotype in the Japanese population. Our key findings were as follows: (1) specific LY75 polymorphisms were significantly associated with CD susceptibility; (2) there were no significant genetic associations between LY75 SNPs and UC susceptibility; and (3) there was a relationship between a specific $L Y 75$ haplotype and a history of bowel resection in patients with CD.

Previous studies have demonstrated that genetic polymorphisms in the MHC region strongly influenced both $\mathrm{CD}$ and UC, with several other SNPs located in non-MHC regions also associated with disease susceptibility. Moreover, genes in the epithelial barrier and the Th17-IL23R pathway have been related to $\mathrm{CD}$ and UC across multiple ethnicities. Since the DEC-205 receptor encoded by LY75 has an important role in T-cell function and homeostasis in the epithelial barrier of the gut, we examined associations between LY75 SNPs and disease susceptibility and uncovered a statistical difference for the rs16822581 SNP by a recessive model between CD patients and controls and for haplotype 2 (GT), which carried the disease susceptibility $\mathrm{T}$ allele at this SNP, indicating that LY75 polymorphisms might be associated 
with CD predisposition in Japan as well. An earlier study demonstrated that a locus in $L Y 75$ was linked to IBD onset [19]. Thus, our findings support shared genetic participation over ethnicities to be involved in IBD etiology, as documented for the Th17-IL23 pathway for CD and UC [17, 18].

A LY75 locus variant has also been associated with erythema nodosum as an extra-intestinal manifestation of CD [31]. However, the lack of patients with this complication in our study made it impossible to conclude any relationship with the LY75 SNPs. As the prevalence of erythema nodosum as an extra-intestinal symptom of CD is $2.3 \%$ [32], larger studies are needed to conclude if $L Y 75$ SNPs are related to this complication in Japanese CD patients.

Interestingly, our study revealed 1 haplotype on the LY75 gene to be associated with the disease phenotype of an absence of surgical history in patients with CD. Some patients required surgical resection due to tissue damage, loss of function, disability, or obstruction within the gut. This haplotype carried the opposite SNPs to disease susceptibility, thus confirming that LY75 function was indeed associated with disease activity in CD.

The current study was preliminary in nature because the numbers of cases and controls were limited. To overcome type I error, larger studies are needed to validate the relationships between LY75 gene polymorphisms and the expression and functions of their gene products.

\section{Conclusions}

Our findings confirm that LY75 SNPs and haplotypes contribute to CD susceptibility and may play a role in disease activity. Further investigation is required to clarify precisely how LY75 functions in the pathogenesis of CD.

\section{Competing Interests}

The authors declare that they have no competing interests.

\section{Acknowledgments}

The authors thank Yuki Akahane and Asami Yamazaki for their technical assistance and Trevor Ralph for his English editorial assistance.

\section{References}

[1] C. Abraham and J. H. Cho, "Inflammatory bowel disease," The New England Journal of Medicine, vol. 361, no. 21, pp. 20662078, 2009 .

[2] E. V. Loftus Jr., "Clinical epidemiology of inflammatory bowel disease: incidence, prevalence, and environmental influences," Gastroenterology, vol. 126, no. 6, pp. 1504-1517, 2004.

[3] M. D. Kappelman, S. L. Rifas-Shiman, K. Kleinman et al., “The prevalence and geographic distribution of Crohn's disease and ulcerative colitis in the United States," Clinical Gastroenterology and Hepatology, vol. 5, no. 12, pp. 1424-1429, 2007.

[4] E. Kuwahara, K. Asakura, Y. Nishiwaki et al., "Effects of family history on inflammatory bowel disease characteristics in
Japanese patients," Journal of Gastroenterology, vol. 47, no. 9, pp. 961-968, 2012.

[5] W. Strober, I. Fuss, and P. Mannon, "The fundamental basis of inflammatory bowel disease," The Journal of Clinical Investigation, vol. 117, no. 3, pp. 514-521, 2007.

[6] U. Pirzer, A. Schönhaar, B. Fleischer, E. Hermann, and K.-H. Meyer zum Buschenfelde, "Reactivity of infiltrating T lymphocytes with microbial antigens in Crohn's disease," The Lancet, vol. 338, no. 8777, pp. 1238-1239, 1991.

[7] D. K. Podolsky, "Inflammatory bowel disease," The New England Journal of Medicine, vol. 347, no. 6, pp. 417-429, 2002.

[8] J. C. Barrett, S. Hansoul, D. L. Nicolae, J. H. Cho, R. H. Duerr, and J. D. Rioux, "Genome-wide association defines more than 30 distinct susceptibility loci for Crohn's disease," Nature Genetics, vol. 40, no. 8, pp. 955-962, 2008.

[9] A. Franke, D. P. B. McGovern, J. C. Barrett et al., "Genome-wide meta-analysis increases to 71 the number of confirmed Crohn's disease susceptibility loci," Nature Genetics, vol. 42, pp. 1118$1125,2010$.

[10] C. A. Anderson, G. Boucher, C. W. Lees et al., "Meta-analysis identifies 29 additional ulcerative colitis risk loci, increasing the number of confirmed associations to 47," Nature Genetics, vol. 43, article 246, 2011.

[11] L. Jostins, S. Ripke, R. K. Weersma et al., "Host-microbe interactions have shaped the genetic architecture of inflammatory bowel disease," Nature, vol. 491, no. 7422, pp. 119-124, 2012.

[12] K. Yamazaki, D. McGovern, J. Ragoussis et al., "Single nucleotide polymorphisms in TNFSF15 confer susceptibility to Crohn's disease," Human Molecular Genetics, vol. 14, no. 22, pp. 3499-3506, 2005.

[13] K. Yamazaki, J. Umeno, A. Takahashi et al., "A genome-wide association study identifies 2 susceptibility loci for Crohn's disease in a Japanese population," Gastroenterology, vol. 144, no. 4, pp. 781-788, 2013.

[14] K. Asano, T. Matsushita, J. Umeno et al., "A genome-wide association study identifies three new susceptibility loci for ulcerative colitis in the Japanese population," Nature Genetics, vol. 41, no. 12, pp. 1325-1329, 2009.

[15] Y. Okada, K. Yamazaki, J. Umeno et al., "HLA-Cw*1202-B* 5201$\mathrm{DRB1}^{*} 1502$ haplotype increases risk for ulcerative colitis but reduces risk for Crohn's disease," Gastroenterology, vol. 141, no. 3, pp. 864-871.e5, 2011.

[16] Y. Ogura, D. K. Bonen, N. Inohara et al., "A frameshift mutation in NOD2 associated with susceptibility to Crohn's disease," Nature, vol. 411, no. 6837, pp. 603-606, 2001.

[17] K. Yamazaki, Y. Onouchi, M. Takazoe, M. Kubo, Y. Nakamura, and A. Hata, "Association analysis of genetic variants in IL23R, ATG16L1 and 5p13.1 loci with Crohn's disease in Japanese patients," Journal of Human Genetics, vol. 52, no. 7, pp. 575-583, 2007.

[18] K. Yamazaki, A. Takahashi, M. Takazoe et al., "Positive association of genetic variants in the upstream region of NKX2-3 with Crohn's disease in Japanese patients," Gut, vol. 58, no. 2, pp. 228232, 2009.

[19] J. Z. Liu, S. van Sommeren, H. Huang et al., "Association analyses identify 38 susceptibility loci for inflammatory bowel disease and highlight shared genetic risk across populations," Nature Genetics, vol. 47, no. 9, pp. 979-986, 2015.

[20] W. Jian, W. J. Swiggard, C. Heufler et al., "The receptor DEC205 expressed by dendritic cells and thymic epithelial cells is involved in antigen processing," Nature, vol. 375, no. 6527, pp. 151-155, 1995. 
[21] W. R. Heath, G. T. Belz, G. M. N. Behrens et al., "Crosspresentation, dendritic cell subsets, and the generation of immunity to cellular antigens," Immunological Reviews, vol. 199, pp. 9-26, 2004.

[22] D. Dudziak, A. O. Kamphorst, G. F. Heidkamp et al., "Differential antigen processing by dendritic cell subsets in vivo," Science, vol. 315 , no. 5808, pp. 107-111, 2007.

[23] H. Soares, H. Waechter, N. Glaichenhaus et al., "A subset of dendritic cells induces $\mathrm{CD} 4^{+} \mathrm{T}$ cells to produce IFN- $\gamma$ by an IL-12-independent but CD70-dependent mechanism in vivo," Journal of Experimental Medicine, vol. 204, no. 5, pp. 1095-1106, 2007.

[24] L. Bonifaz, D. Bonnyay, K. Mahnke, M. Rivera, M. C. Nussenzweig, and R. M. Steinman, "Efficient targeting of protein antigen to the dendritic cell receptor DEC-205 in the steady state leads to antigen presentation on major histocompatibility complex class I products and peripheral CD8 ${ }^{+} \mathrm{T}$ cell tolerance," The Journal of Experimental Medicine, vol. 196, no. 12, pp. 16271638, 2002.

[25] T. Fukaya, R. Murakami, H. Takagi et al., "Conditional ablation of $\mathrm{CD} 205^{+}$conventional dendritic cells impacts the regulation of T-cell immunity and homeostasis in vivo," Proceedings of the National Academy of Sciences of the United States of America, vol. 109, no. 28, pp. 11288-11293, 2012.

[26] G. Van Assche, A. Dignass, J. Panes et al., “The second European evidence-based consensus on the diagnosis and management of Crohn's disease: definitions and diagnosis," Journal of Crohn's and Colitis, vol. 4, article 7, 2010.

[27] A. Dignass, R. Eliakim, F. Magro et al., "Second European evidence-based consensus on the diagnosis and management of ulcerative colitis part 1: definitions and diagnosis," Journal of Crohn's and Colitis, vol. 6, no. 10, pp. 965-990, 2012.

[28] M. Ota, H. Fukushima, J. K. Kulski, and H. Inoko, "Single nucleotide polymorphism detection by polymerase chain reaction-restriction fragment length polymorphism," Nature Protocols, vol. 2, no. 11, pp. 2857-2864, 2007.

[29] J. C. Barrett, B. Fry, J. Maller, and M. J. Daly, "Haploview: analysis and visualization of LD and haplotype maps," Bioinformatics, vol. 21, no. 2, pp. 263-265, 2005.

[30] S. B. Gabriel, S. F. Schaffner, H. Nguyen et al., "The structure of haplotype blocks in the human genome," Science, vol. 296, no. 5576, pp. 2225-2229, 2002.

[31] A. Alonso, E. Domènech, A. Julià et al., "Identification of risk loci for crohn's disease phenotypes using a genome-wide association study," Gastroenterology, vol. 148, no. 4, pp. 794-805, 2015.

[32] T. R. Card, S. M. Langan, and T. P. Chu, "Extra-gastrointestinal manifestations of inflammatory bowel disease may be less common than previously reported," Digestive Diseases and Sciences, vol. 61, no. 9, pp. 2619-2626, 2016. 


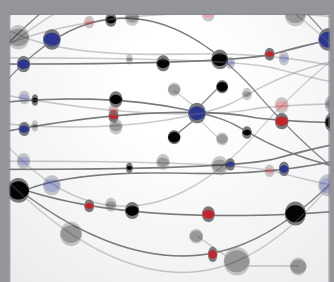

The Scientific World Journal
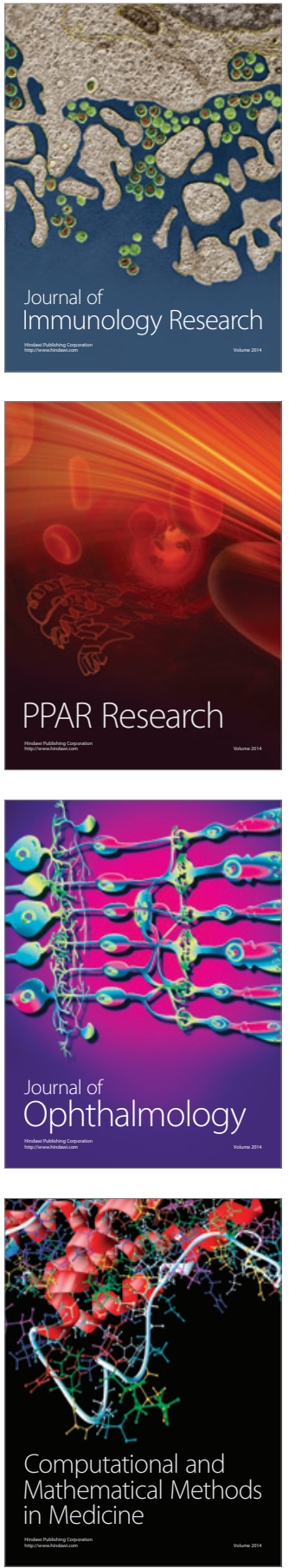

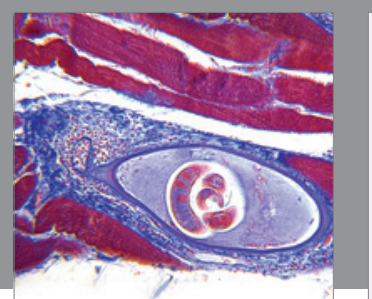

Gastroenterology Research and Practice

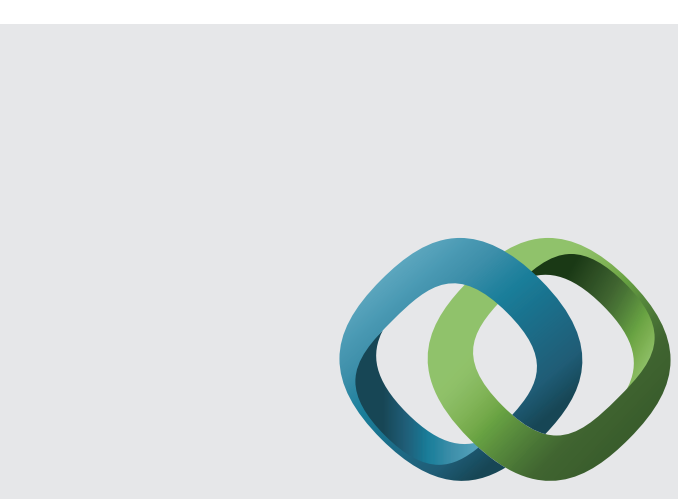

\section{Hindawi}

Submit your manuscripts at

http://www.hindawi.com
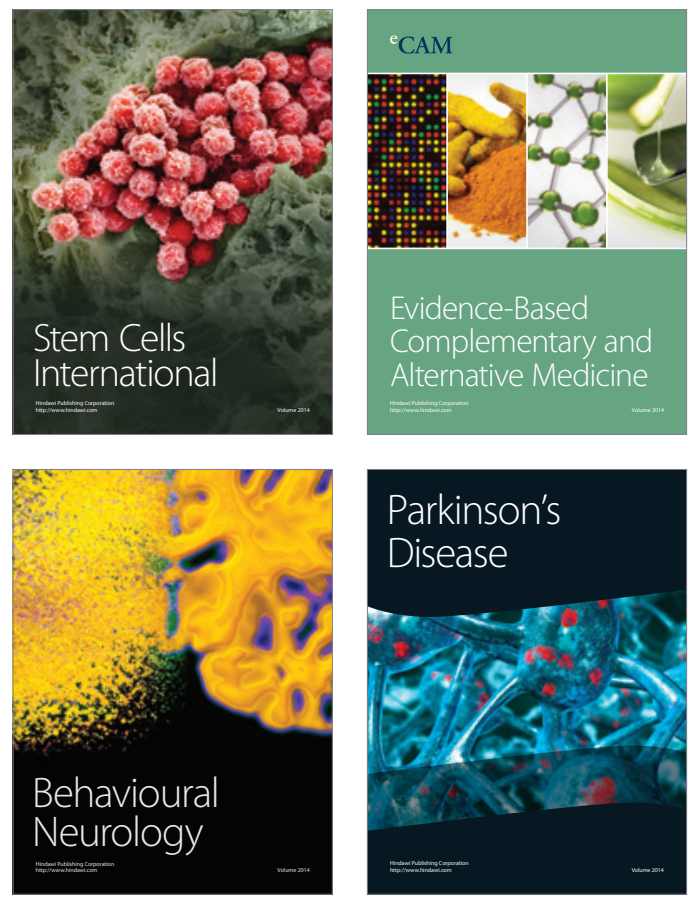
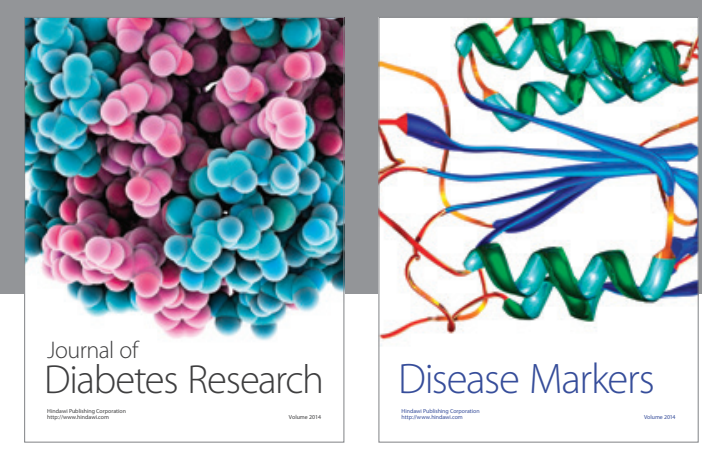

Disease Markers
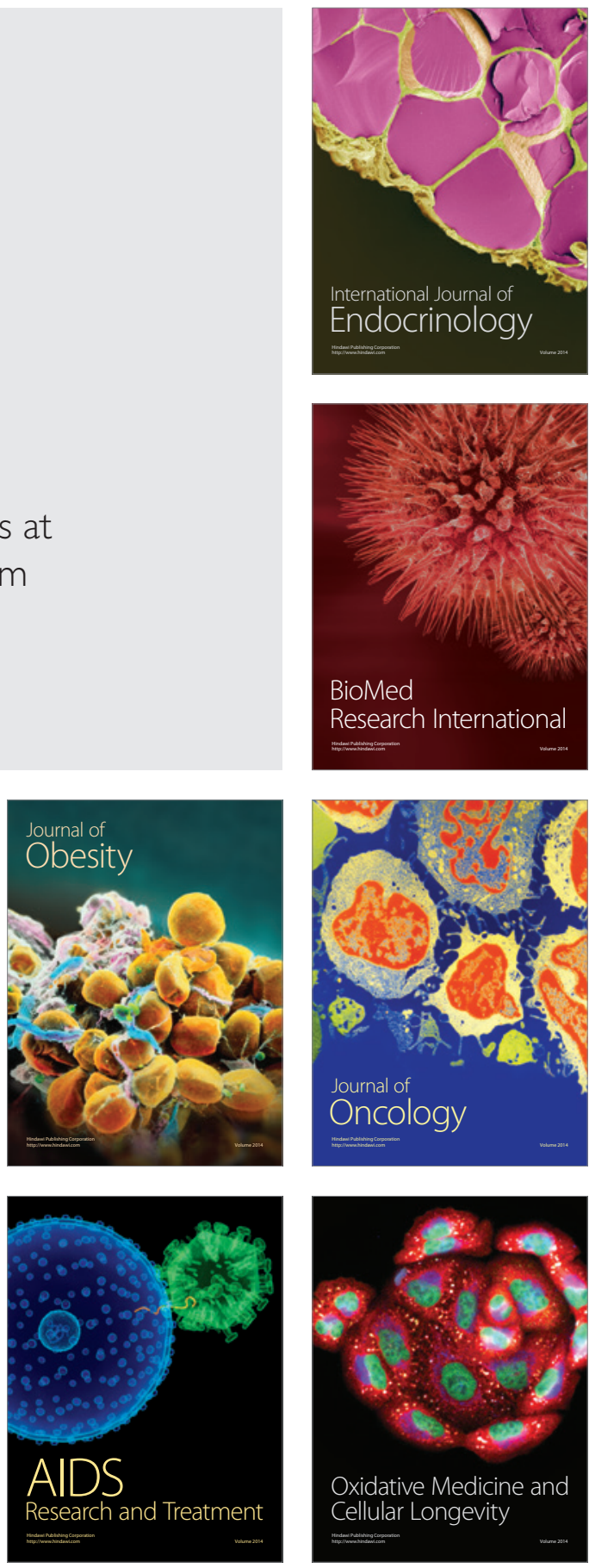